\title{
Prevalência de Sintomas Depressivos em uma Amostra de Prostitutas de Porto Alegre
}

\author{
Lucas Schreiner* \\ Leonardo Ludwig Paim* \\ Fabiano Ramos* \\ Edson Vieira Cunha Filho* \\ Diogo Marílio Martins*
}

\author{
Claudio Lopes Silva Junior* \\ Marcelo Coutinho Baú* \\ Tiago Madeira Cardinal* \\ Nina Rosa Furtado** \\ Patrícia Picon ${ }^{\star \star *}$
}

\section{INTRODUÇÃO}

Os "favores femininos" são reconhecidos como um dos mais remotos produtos de comércio da humanidade. Desde a Grécia Antiga, tem-se relatos da existência da prostituição como uma atividade profissional que, ao longo da história, de maneira mais ou menos intensa, sofreu restrições e foi situada à margem da sociedade. ${ }^{1}$

Na cidade de Porto Alegre, existem inúmeras prostitutas que trabalham sob as mais diversas condições em bares, boates e nas ruas. De acordo com a legislação brasileira, a prostituição não constitui um delito, uma vez que "a

\footnotetext{
* Doutorandos da Faculdade de Medicina da Pontifícia Universidade Católica do Rio Grande do Sul (FAMED/PUCRS).

** Psiquiatra, Mestre em Comunicação Social pela PUCRS, Psicanalista da SPPA (IPA), Professora Assistente do Departamento de Psiquiatria e Medicina Legal da FAMED/PUCRS.

*** Psiquiatra, Mestre em Epidemiologia pela Harvard School of Public Health, Professora Assistente do Departamento de Psiquiatria e Medicina Legal da FAMED/PUCRS.

Instituição: Departamento de Psiquiatria e Medicina Legal da Faculdade de Medicina da Pontifícia Universidade Católica do Rio Grande do Sul FAMED PUCRS, Porto Alegre, RS, Brasil.
}

todo cidadão se permite a liberdade de disposição do próprio corpo, sendo passíveis de punição os casos de exploração da prostituição". ${ }^{2}$

A depressão, cujos sintomas centrais segundo os critérios da Classificação Internacional de Doenças da Organização Mundial da Saúde (CID -10) ${ }^{3}$, são o humor deprimido, a perda de interesse e o prazer e a energia reduzida, tem sido registrada desde a antigüidade, assim como a prostituição.

Entre 1990 e 1992, o "National Comorbidity Survey" (NCS) ${ }^{4}$ realizado em amostra probabilística da população norte-americana, de 15 a 54 anos de idade, utilizando os critérios diagnósticos da DSM III-R, revelou prevalências, entre as mulheres, de episódio depressivo maior de $12,9 \%$ em um ano e $21,3 \%$ para toda vida. Neste estudo, os fatores de risco para quaisquer transtornos mentais em um ano foram: ser do gênero feminino, com idade entre 15 e 24 anos, com baixa renda e baixo nível de escolaridade. Além disso, o transtorno depressivo maior acomete mais freqüentemente indivíduos separados ou divorciados, ou seja, que não mantenham relações interpessoais íntimas duradouras. Presume-se que tais fatores de 
risco estejam presentes em populações de prostitutas de diferentes países e em especial em paises com o perfil econômico do Brasil.

Em 1998, a Organização Mundial da Saúde (OMS) estabeleceu um projeto ambicioso para um estudo trans-nacional de prevalência de transtornos mentais o "WHO - International Consortium in Psychiatric Epidemiology" (WHOICPE). O WHO-ICPE utilizou o CIDI (Composite International Diagnostic Interview), o qual tem sido largamente testado em estudos populacionais de diferentes línguas. Em 2000, foram divulgados os resultados da primeira fase do WHO-ICPE com dados de prevalência para toda a vida de sete países, incluindo o Brasil, em amostras probabilísticas populacionais, num total de 29644 indivíduos estudados. $\mathrm{Na}$ fase brasileira do WHO-ICPE, o estudo foi realizado em amostra probabilística de 1464 indivíduos adultos de São Paulo. As prevalências de transtornos de humor ao longo da vida, em um ano e em um mês, foram respectivamente de 15,5; 7,1 e $4,9 \%$. Nos sete países em que o estudo foi realizado, os transtornos mentais são de inicio precoce e com idade média de 26 anos para os transtornos de humor 5 .

Existem poucos estudos relacionando sintomas ou transtornos depressivos e prostituição, e nenhum estudo realizado em Porto Alegre foi encontrado pelos autores. Este estudo tem por objetivo avaliar a presença de sintomas depressivos em uma amostra de prostitutas ligadas a uma organização não governamental brasileira localizada em Porto Alegre, e sua associação com possíveis fatores de riscos.

\section{MATERIAL E MÉTODOS}

No presente estudo foi definido como prostituta toda a mulher que declara praticar a prostituição. A prostituição tem sido definida como a atividade institucionalizada que visa a ganhar dinheiro com a cobrança por atos sexuais e a exploração de prostitutas. ${ }^{6} \mathrm{~A}$ população em estudo foi composta por todas as mulheres prostitutas que buscaram a organização na governamental Núcleo de Estudo da Prostituição (NEP), no período de outubro de 2000 a janeiro do ano de 2001.

Através de delineamento transversal, em amostra não aleatória consecutiva de mulheres, entre 18 e 60 anos de idade, após assinatura de consentimento informado, garantia de sigilo dos dados e de anonimato, os entrevistadores avaliaram os seguintes dados demográficos e sócio-econômicos: idade, naturalidade, procedência, companheiro fixo, escolaridade, reli- gião, prática religiosa, cor da pele, local de trabalho, razão para manter a atividade, renda média mensal, intenção de parar de prostituirse, contato com filhos, abortos, uso de preservativos, doenças sexualmente transmissíveis ao longo da vida, tabagismo, uso de álcool e de drogas ilícitas.

Na seqüência, os sintomas depressivos foram mensurados através do Inventário para depressão de Beck $(\mathrm{BDI})^{7}$, instrumento de autopreenchimento composto por 21 itens que avaliam a sintomatologia presente na última semana. Os investigadores foram orientados para a coleta padronizada de dados através de protocolo de pesquisa elaborado pelos autores e para a aplicação do BDI.

O BDI foi extensamente validado em amostras clínicas e populacionais brasileiras por $\mathrm{Cu}$ nha e colaboradores ${ }^{8}$, que encontrou os seguintes pontos de coorte para diferentes intensidades dos sintomas depressivos: mínimo (0-11), leve (12-19), moderado (20-35) e grave (36 a 63$)$.

No presente estudo, foi utilizado o ponto de corte maior ou igual a 13 para identificação de casos de indivíduos portadores de sintomas depressivos, através da aplicação do BDI, utilizado por Lasa e colaboradores ${ }^{9}$ em estudo de base populacional para rastreamento de transtornos depressivos, com excelente perfil psicométrico.

Todos os indivíduos da amostra apresentavam escolaridade acima da quarta série do ensino fundamental e foram submetidos à aplicação do Inventário para Depressão de Beck, sem interferência dos investigadores.

Os dados foram tabulados e analisados através do programa estatístico SPSS versão 10.0. ${ }^{10} \mathrm{~A}$ análise descritiva fornece freqüência, média e desvio padrões, e a análise bivariada foi realizada através dos testes Qui-quadrado e Teste exato de Fisher.

O estudo foi previamente aprovado pelo Comitê de Ética em Pesquisa do Hospital São Lucas da Pontifícia Universidade Católica do Rio Grande do Sul de Porto Alegre.

\section{RESULTADOS}

A amostra foi composta de 97 mulheres prostitutas, e a análise descritiva das variáveis demográficas e sócio-econômicas é apresentada na tabela1.

$\mathrm{Na}$ amostra, o escore médio do Inventário para Depressão de Beck foi de 19,1 $(\mathrm{dp}=10,9)$ e $67 \%$ das entrevistadas apresentam pontuação maior ou igual a 13, classificadas portanto 
Tabela 1. Descrição da amostra de 97 prostitutas de Porto Alegre

\begin{tabular}{ll}
\hline CARACTERÍSTICA & RESULTADO \\
\hline Idade Média & 29,6 anos (dp=8,5 anos) \\
\hline Naturalidade & $\begin{array}{c}51 \text { indivíduos }(52,6 \%) \text { naturais da Região } \\
\text { Metropolitana de Porto Alegre }\end{array}$ \\
\hline Procedência & 62 indivíduos $(63,9 \%)$ procedente de Porto Alegre \\
\hline Companheiro fixo & 52 indivíduos $(53,6 \%)$ possuem companheiro \\
\hline Escolaridade & 45 indivíduos $(46,4 \%)$ entre $4^{a}$ e $8^{a}$ série \\
\hline Religião & 67 indivíduos $(69,1 \%)$ são católicos \\
\hline Relato de prática religiosa & 26 indivíduos $(27,1 \%)$ consideram-se praticantes \\
\hline Cor da pele & 64 indivíduos $(66,6 \%)$ são brancos \\
\hline Local de trabalho & 36 indivíduos $(37,1 \%)$ trabalham em salas de \\
& prostituição \\
\hline Razão para continuar a atividade & 89 indivíduos $(91.8 \%)$ razões econômicas \\
\hline Renda média mensal & R\$ 1001,1 \\
\hline Intenção de parar de prostituir-se & 84 indivíduos $(86,6 \%)$ pensam em deixar a profissão \\
\hline Contato com filhos & 90 indivíduos $(92,3 \%)$ tem contato com pelo menos \\
& um filho \\
\hline Abortos & 47 indivíduos $(48,5 \%)$ já tiveram algum aborto \\
\hline Preservativos & 91 indivíduos $(93,8 \%)$ sempre usam preservativos \\
\hline Doenças sexualmente transmissíveis (DST) & 28 indivíduos $(28,9 \%)$ já tiveram alguma DST \\
\hline Tabagismo & 55 indivíduos $(56,7 \%)$ fumam \\
\hline Uso de álcool & 60 indivíduos $(61,9 \%)$ usam bebida alcoólica \\
\hline Drogas ilícitas & 10 indivíduos $(10,3 \%)$ usam drogas atualmente \\
\hline &
\end{tabular}

Tabela 2. Prevalência de Sintomas depressivos $(n=97)$

\begin{tabular}{lll} 
Nível de sintomas & $\mathrm{n}$ & $\%$ \\
\hline Sintomas mínimos $(0-11)$ & 27 & $27,8 \%$ \\
\hline Sintomas leves (12-19) & 24 & $24,7 \%$ \\
\hline Sintomas moderados (20-35) & 39 & $40,2 \%$ \\
\hline Sintomas graves (36-63) & 7 & $7,2 \%$ \\
\hline BDI ponto de corte $>$ ou $=13$ & 65 & $67 \%$ \\
\hline
\end{tabular}

como portadoras de sintomas depressivos. Entre as entrevistadas $24,7 \%$ apresentaram sintomas leves, 40,2\% sintomas moderados e 7,2 $\%$ sintomas graves. (Ver tabela 2).

No grupo de entrevistadas com sintomas depressivos, 70,7\% eram usuárias de álcool e $38,4 \%$ tinham história prévia de doenças sexualmente transmissíveis com associação estatisticamente significativa entre estas variáveis e a presença de sintomas depressivos. (ver gráficos 1 e 2). Ainda no grupo com sintomas depressivos, 32,2\% relatavam praticar alguma religião com associação estatisticamente signi- ficativa entre a presença de sintomas depressivos e a ausência de prática religiosa. (ver gráfico 3)

Nas demais análises bivariadas, não foram detectadas associações estatísticamente significativas entre a presença de sintomas depressivos e idade, naturalidade, procedência, companheiro fixo, escolaridade, religião, cor da pele, local de trabalho, razão para manter a atividade, renda média mensal, intenção de parar de prostituir-se, contato com filhos, abortos, uso de preservativos, tabagismo e uso de drogas ilícitas. 
Gráfico 1: Uso de Álcool x Sintomas Depressivos

Quantidade de

indivíduos

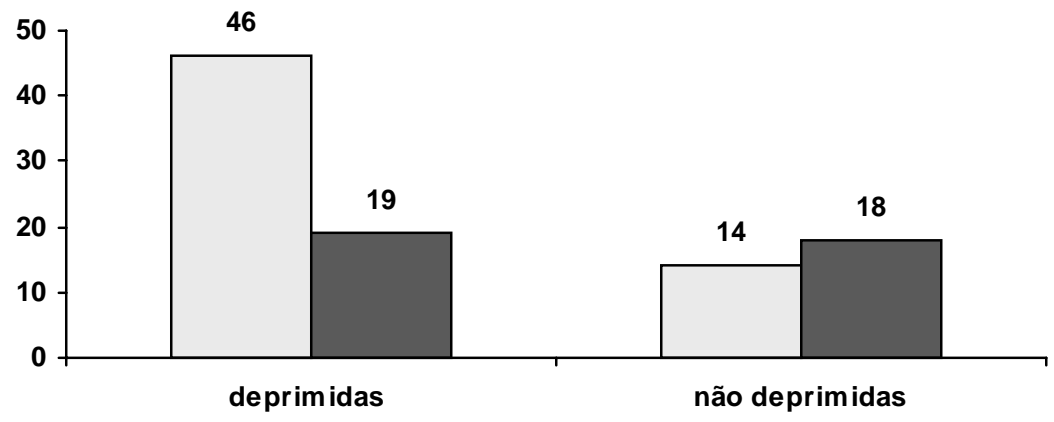

$\square$ uso de álcool

$\square$ sem uso de álcool

RR 1,49 ( IC 95\% 1,04 -2,10); p= 0,01; teste Qui-quadrado

Gráfico 2: História de Doenças Sexualmente Transmissíveis x Sintomas Quantidade de indivíduos

Depressivos

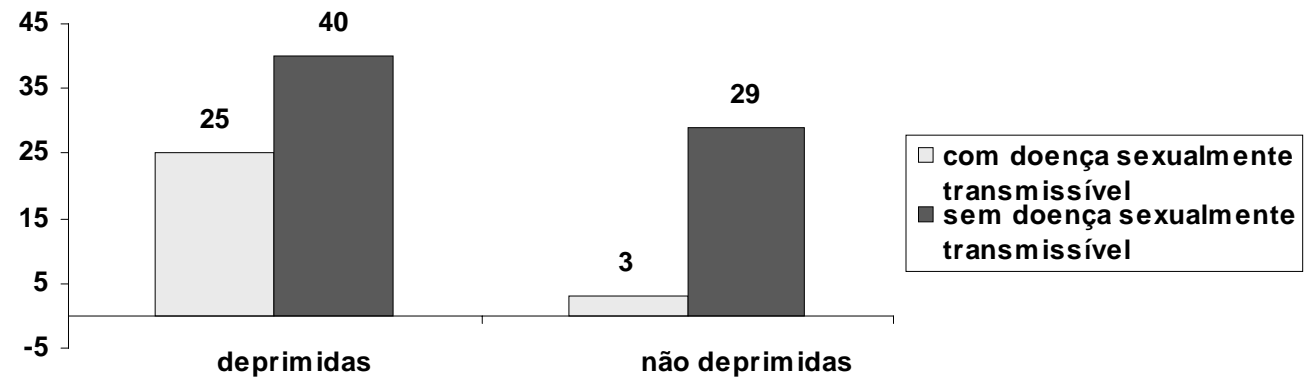

RR 1,54 (C 95\% 1,21- 1,95); $p=0,002$; teste exato de Fisher

Gráfico 3: Relato de Prática Religiosa x Sintomas Depressivos

Quantidade de indivíduos

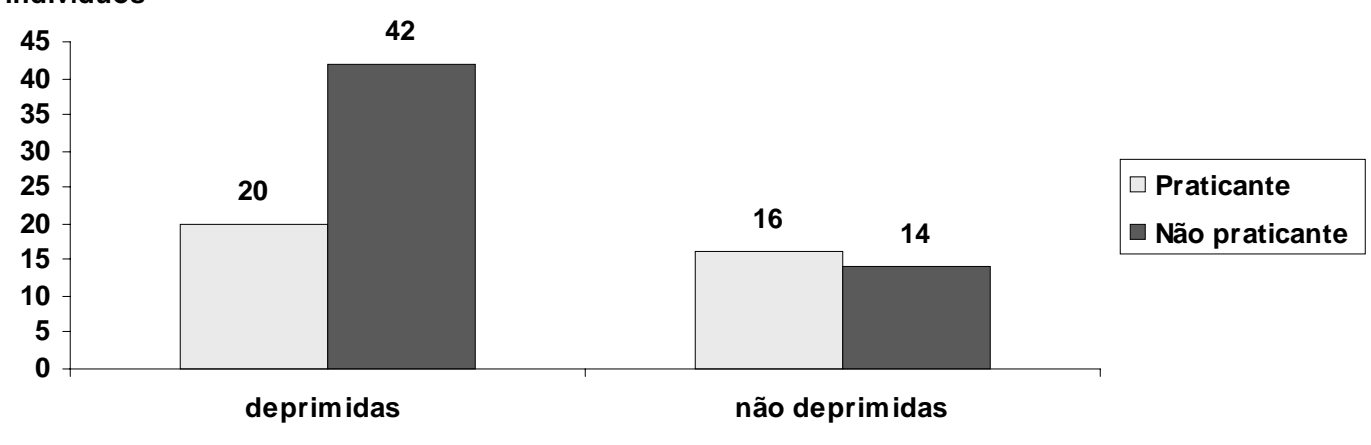

RR 0,74 (C 95\% 0,53-1,03); $p=0,044$; teste Qui-quadrado 


\section{DISCUSSÃO}

A prevalência de sintomatologia depressiva em $67 \%$ dos indivíduos da amostra chama a atenção, bem como a associação estatisticamente significativa entre a presença destes sintomas com uso de álcool, história de doenças sexualmente transmissíveis e ausência de prática religiosa. A prevalência de sintomas depressivos na população feminina de Porto Alegre ainda não foi avaliada. Entretanto, estudo epidemiológico populacional ${ }^{11}$ realizado na década de 90, em amostras probabilísticas representativas de três capitais brasileiras, utilizando o Inventário de Sintomas do DSM-III (IS-DSM-III) revelou uma taxa de prevalência em um ano de $14,5 \%$ de transtornos depressivos na população feminina de Porto Alegre.

Alegria e colaboradores ${ }^{12}$ estudaram a associação entre a presença do vírus HIV, comportamento de risco e sintomas depressivos, avaliados através do "Center for Epidemiologic Studies Depression Scale", instrumento que avalia sintomatologia depressiva, em 127 prostitutas de Porto Rico. O estudo revelou uma prevalência de $70 \%$ de sintomas depressivos. Apesar da amostra porto-riquenha estar exposta a outros riscos que não o ocupacional e utilizar instrumento distinto para avaliar a prevalência de sintomas depressivos, o resultado foi semelhante ao do presente estudo.

A prevalência ponto de $67 \%$ de sintomas depressivos na presente amostra pode estar relacionada ao fato do Inventário para depressão de Beck ser um instrumento de rastreamento, com características psicométricas que privilegiam a sensibilidade. ${ }^{13}$ Entretanto, convém lembrar que o BDI possui boas especificidade e sensibilidade quando se utiliza o ponto de corte de treze pontos, como demonstrado por Lasa e colaboradores $^{9}$ em estudo de base populacional, em amostra aleatória de 1250 indivíduos entre 18 e 64 anos de ambos os gêneros, para avaliação do BDI como instrumento de rastreamento para transtornos depressivos. Neste estudo, os indivíduos que apresentavam 13 ou mais pontos foram submetidos a avaliação por psiquiatras utilizando o "Schedules for Clinical Assesment in Neuropsychiatry" (SCAN) como padrão ouro. O estudo realizado na Espanha demonstrou que o ponto de corte maior ou igual a 13 apresenta $100 \%$ de sensibilidade, $99 \%$ de especificidade, valor preditivo positivo de $72 \%$ e valor preditivo negativo de $1 \%$. Há evidências, portanto, de que o ponto de corte proposto por Lasa e seus colaboradores é útil para detecção de prováveis casos de transtornos de- pressivos em população não clínica, com acurácia global do BDI de $98 \%$.

Em estudo utilizando o BDI para avaliação de sintomas depressivos em amostra de estudantes de medicina chinesas em Honk Kong ${ }^{14}$, usando o ponto de corte igual ou maior que 9, foi observada uma prevalência menor que $50 \%$. No presente estudo, se fosse utilizado o ponto de corte de 9, a prevalência ponto de sintomas depressivos na amostra de prostitutas sería de $82 \%$.

Cunha e colaboradores ${ }^{8}$, em seus estudos de validação em amostras brasileiras do Inventário de depressão de Beck, relatam as prevalências de diferentes intensidades de sintomas depressivos em uma amostra de 212 pacientes portadores de episódio depressivo maior, de ambos os gêneros, realizado em Porto Alegre com nível mínimo 5,7\%; nível leve 15,8 \%; nível moderado $45,5 \%$ e nível grave $33 \%$. A amostra de prostitutas aqui estudada apresenta uma prevalência de sintomas de nível leve de $24,7 \%$, de nível moderado de $40,2 \%$ e de nível grave de $7,2 \%$.

Em relação ao uso de álcool, $61,9 \%$ das entrevistadas faz uso de bebidas alcoólicas de forma rotineira. A prevalência em 1 ano de transtornos mentais e de comportamento decorrentes do uso de álcool em estudo populacional realizado na população de Porto Alegre por Busnello e colaboradores ${ }^{15}$, utilizando o Inventário de Sintomas do DSM-III (IS-DSM-III), foi de $2,7 \%$ entre as mulheres. Embora a investigação da prevalência de abuso ou dependência ao álcool não tenha sido o objetivo do presente estudo, limitando portanto a comparação dos dados com os de outros estudos, salientase que as mulheres aqui estudadas apresentam uma alta prevalência de uso de álcool.

A prevalência de $28,9 \%$ de doenças sexualmente transmissíveis ao longo da vida, número aparentemente reduzido dados os riscos inerentes à pratica profissional da amostra, pode ser parcialmente explicado pela taxa de $93,8 \%$ de utilização de preservativos encontrada. Estes achados podem estar relacionados ao trabalho desenvolvido pela organização não governamental Núcleo de Estudo da Prostituição (NEP) onde as entrevistas foram realizadas, que inclui em sua atuação palestras sobre esses temas e a distribuição de preservativos às profissionais que comparecem ao local. Aqui cabe ressaltar também a observação de que as prostitutas responderam a essas questões em relação aos clientes e não aos seus companheiros fixos. Um estudo prévio ${ }^{16}$ feito com prostitutas de Porto Alegre revelou que o preservati- 
vo serve como um diferenciador de relacionamentos. A maioria das mulheres considera um sinal de fidelidade ao marido ou companheiro fixo a não utilização de preservativos com ele, e o preservativo simbolizaria a diferença entre a vida particular e a profissional.

Quanto ao relato de prática religiosa, 27\% praticam alguma religião e um grande número de mulheres, apesar de declarar uma religião, não a pratica. A prática religiosa aparece na amostra como um fator de proteção em relação à presença de sintomas depressivos, o que corresponde a achados de literatura em que a prática religiosa está associada com a redução de risco de transtornos depressivos ao longo da vida e a melhoria de qualidade de vida. ${ }^{17,18}$

Embora a maioria das entrevistadas referissem desejo de abandonar a profissão, 91,8\% delas relataram que a motivação para seguir com sua prática profissional é a compensação financeira.

Assim, foi verificada uma alta taxa de prevalência ponto de sintomas depressivos na amostra estudada, com níveis moderado a grave em quase metade dos casos de mulheres prostitutas com sintomatologia depressiva. A luz da revisão de literatura apresentada é razoável levantar-se a hipótese de ser este um grupo de risco para transtornos depressivos. Além disto, análises bivariadas mostraram associação estatisticamente significativa entre a presença de sintomas depressivos (ponto de coorte igual ou maior que 13) e uso de álcool, história de doenças sexualmente transmissíveis e falta de algum tipo de prática religiosa. Análise multivariada não foi realizada em razão do tamanho amostral.

\section{CONCLUSÕES}

$\mathrm{Na}$ amostra estudada, foi encontrada uma alta prevalência ponto de sintomas depressivos (67\%), na qual $47,4 \%$ das entrevistadas apresentaram sintomatologia moderada a grave. Associações estatisticamente significativas entre a presença de sintomas depressivos com uso de álcool, história de doenças sexualmente transmissíveis e ausência de prática religiosa foram verificadas em análise bivariada.

A presença de sintomas depressivos pode ser um indicativo de transtorno depressivo maior, mas pode também corresponder a outros transtornos depressivos como: distmia, reações de ajustamento com sintomas depressivos, episódio depressivo em transtorno de humor bipolar e outros transtornos mentais. $O$ cluir entrevista clínica psiquiátrica diagnóstica, não sendo objeto do presente estudo. Entretanto, o excelente perfil psicométrico do BDI para rastreamento de transtornos depressivos em população geral sugere fortemente a presença destes quadros entre as prostitutas estudadas.

$O$ estudo tem o objetivo de chamar a atenção para essa população de risco colocada à margem da sociedade, mas que, seguramente, necessita de atenção e investigações que complementem e enriqueçam os dados aqui relatados sobre este grupo sobre o qual pouco se sabe e se pesquisa em nosso meio.

Os resultados da amostra estudada sugerem ser esta uma população de risco para transtornos depressivos e mais estudos são necessários sobre a população de prostitutas de Porto Alegre e em diferentes amostras de outras cidades brasileiras.

\section{REFERÊNCIAS BIBLIOGRÁFICAS}

1. Bassermann L. História da Prostituição: Uma Interpretação Cultural. Rio de Janeiro: Civilização Brasileira; 1968.

2. Assis A, editor. A Prostituição em Debate: Depoimentos, Análises, Procura de Soluções. São Paulo: Paulinas; 1982.

3. Organização Mundial da Saúde. Classificação de Transtornos Mentais e de Comportamento da CID-10: Descrições Clínicas e Diretrizes Diagnósticas. Porto Alegre: Artes Médicas; 1993. p. 117-126.

4. Kessler RC, McGonagle KA, Zhao S, Nelson CB, Hughes $M$, Eshleman $S$ et al. Lifetime and 12-month prevalence of DSM-III-R psychiatric disorders in the United States. Results from the National Comorbidity Survey. Arch. Gen. Psychiatric 1994; 51: 8-18.

5. Picon P. Epidemiologia Psiquiátrica. In: Cataldo Neto A, Gauer GC e Furtado NR, editores. Psiquiatria para estudantes de medicina. Porto Alegre: EDIPUCRS; 2003, p. 83-90.

6. Houaiss A, Villar MS, Franco FMM. Dicionário Houaiss da Língua Portuguesa. Rio de Janeiro: Ed. Objetiva; 2001, p. 2316

7. Beck AT, Steer RA. Beck Depression Inventory- Manual. San Antonio: The Psychological Corporation; 1987, p.124.

8. Cunha JA. Manual da Versão em português das Escalas Beck. São Paulo: Casa do Psicólogo Livraria e Editora; 2001, p.1-171.

9. Lasa L, Ayuso-Mateos JL, Vazquez- Barquero JL, DiezManrique FJ, Dowrick CF. The use of the Beck Depression Inventory in the general population: a preliminary analysis. J Affect Disor 2000; 57: 261-5.

10. SPSS Incorporated. SPSS for Windows - version 10.0. Chicago IL: SPSS; 2000.

11. Almeida Filho N, Mari JJ, Coutinho E, Franca JF, Fernandes J, Andreoli SB, Busnello ED. Brazilian mulicentric study of psychiatric morbidity. Methodological features and prevalence estimates. $\mathrm{Br} J$ Psychiatry 1997; 171: 524-9.

12. Alegria $M$, Vera $M$, Freeman $D H$ Jr, Robles $R$, Santos $M C$, Rivera CL. Hiv infection, risk behaviors and depressive symptoms among Puerto Rican sex workers. Am J Public Health 1994; 84: 2000-2.

13. Fachel JMG, Camey S. Avaliação psicométrica: a quali- 
dade das medidas e o entendimento dos dados. In: $\mathrm{Cu}$ nha JA, editora. Psicodiagnóstico. $5^{\mathrm{a}}$ edição. Porto Alegre: Artemed; 2000. p. 158-170.

14. Han L, Wang K, Du Z, Cheng Y, Simons JS, Rosenthal NE. Seasonal variations in mood and behavioral among chinese medical students. Am J Psychiatry 2000; 157 133-5.

15. Busnello ED, Pereira MO, Knapp WP, Salgado CAI, Taborda JGV, Knijnik L, et al. Morbidade Psiquiátrica na população urbana de Porto Alegre. J Bras Psiq 1992; 41: 507-512.

16. Fábregas-Martinês Al, Benedetti MR. Na batalha: Sexualidade, identidade e poder no universo da prostituição. Porto Alegre: Da Casa; 2000.

17. Kendler KS, Liu XQ, Gardner CO, McCullough ME, Larson D, Prescott CA. Dimension of religiosity and their relationship to life time psychiatric and substance use disorders. Am J Psychiatry 2003;160: 469- 503.

18. Rocha NS, Fleck M. Health status and quality of life- the effect of spirituality, religiosity and personal believes. Rev Bras Psiquiatr 2002; 24 (supl II): 130.

\section{RESUMO}

Introdução: a prevalência de transtornos depressivos na população feminina de Porto Alegre é estimada em $14,5 \%$. Não existem relatos sobre a prevalência de sintomas ou transtornos depressivos entre as prostitutas, população de risco para transtornos mentais.

Objetivos: quantificar a prevalência de sintomas depressivos em amostra de prostitutas de Porto Alegre e fatores associados.

Material e Métodos: uma amostra consecutiva e não aleatória de 97 mulheres entre 18 e 60 anos, cadastradas na Organização Não Governamental Núcleo de Estudos da Prostituição (NEP), de Porto Alegre, foi estudada. Após consentimento informado, as entrevistadas foram investigadas através do Inventário para depressão de Beck (BDI). O ponto de corte igual ou maior que 13 foi utilizado para detecção de sintomas depressivos.

Resultados: na amostra estudada, a idade média foi de 29,6 anos (dp 8,5 anos); 67\% apresentaram sintomas depressivos (ponto de corte ${ }^{3} 13$ ) com escore médio no $B D I$ de 19,1 (dp 10,9); 24,7\% da amostra apresentava sintomas leves; $40,2 \%$ sintomas moderados e $7,2 \%$ sintomas graves. Houve associação estatisticamente significativa entre a presença de sintomas depressivos e uso de álcool, história de doenças sexualmente transmissíveis e ausência de prática religiosa $(p<0,05)$

Conclusão: além de alta taxa de prevalência de sintomas depressivos (67\%), 47,4\% das mulheres avaliadas apresentaram níveis moderado e grave de sintomatologia. O uso de álcool, história de doenças sexualmente transmissíveis e ausência de prática religiosa surgiram como fatores associados à presença de sintomas depressivos na amostra estudada.

Descritores: Prostituição, depressão, transtornos depressivos.

\section{ABSTRACT}

Introduction: the prevalence of depressive disorders in the female population in Porto Alegre was estimated to be $14.5 \%$. There are no reports on the prevalence of depressive symptoms or depressive disorders among prostitutes that are a group at risk for mental disorders.

Objectives: the main purpose of this study was to evaluate the prevalence of depressive symptoms and to identify associated factors in a sample of Porto Alegre prostitutes.

Material and Methods: a consecutive and nonrandomized sample of 97 women between 18 and 60 years, registered at a non-governmental organization in Porto Alegre was studied using the Beck Depression Inventory (BDI) after informed consent. A cut off point equal or higher then 13 was used to detect depressive symptoms

Results: the mean age $( \pm S D)$ was $29.6(8,5)$, depressive symptoms (cut off ${ }^{3} 13$ ) were noted in $67 \%$ of the sample, the mean score $( \pm S D)$ of $B D I$ was 19.1 (10.9). The depressive symptoms were found to be mild in $24.7 \%$, moderate in $40.2 \%$ and severe in $7.2 \%$ of the sample. A statistically significant association was found between depressive symptoms and use of alcohol, and sexually-transmitted diseases, and lack of religious practices $(p<0,05)$.

Conclusion: besides the high prevalence of depressive symptoms (67\%) $47.4 \%$ of the women studied presented moderate or severe levels of depression. Use of alcohol, and sexually-transmitted diseases and lack of religious practices were associated with depressive symptoms in the studied sample.

Keywords: Prostitution, depression, depressive disorders.

Title: Prevalence of Depressive Symptoms in a Sample of Prostitutes in Porto Alegre

\section{RESUMEN}

Introducción: la prevalencia de trastornos depresivos en la población femenina de Porto Alegre es estimada en $14,5 \%$. No existen relatos sobre la prevalencia de síntomas o trastornos depresivos entre las prostitutas, población de riesgo para trastornos mentales.

Objetivos: cuantificar la prevalencia de síntomas depresivos en muestra de prostitutas de Porto Alegre y factores asociados.

Material y Métodos: fue estudiada una muestra consecutiva y no aleatoria de 97 mujeres entre 18 y 60 años, cadastradas en la Organización No Governamental Nucleo de Estudios de la Prostitución (NEP), de Porto Alegre. Después de informado el consentimiento, las entrevistadas fueron investigadas a través del Inventario para Depresión de Beck $(B D I)$. El punto de corte igual o mayor que 13 fue utilizado para detección de síntomas depresivos. 
Resultados: en la muestra estudiada la edad media fue de 29,6 años (dp 8,5 años), 67\% presentaron síntomas depresivos (punto de corte ${ }^{3}$ 13) con escore medio en el BDI de 19,1 (dp 10,9); $24,7 \%$ de la muestra presentaba síntomas leves, 40,2 \% síntomas moderados y 7,2 \% síntomas graves. Hubo asociación estatísticamente significativa entre la presencia de síntomas depresivos y el uso de alcohol, histórico de enfermedades sexualmente transmisibles y la ausencia de práctica religiosa, $(p<0,05)$.

Conclusión: además de la alta tasa de prevalencia de síntomas depresivos (67\%) 47,4\% de las mujeres evaluadas presentaron niveles moderado y grave de sintomatología. El uso de alcohol, histórico de enfermedades sexualmente transmisibles y la ausencia de práctica religiosa surgieron como factores aso- ciados a la presencia de síntomas depresivos en la muestra estudiada.

Palabras-clave: Prostitucion, depression, transtornos depressivos.

Título: Prevalencia de Síntomas Depresivos en una Muestra de Prostitutas de Porto Alegre

Endereço para correspondência:

Patrícia Picon

Rua Padre Chagas 415/ 803

CEP 90570-080

Porto Alegre, RS, Brasil

E-mail: ppicon@terra.com.br 